\title{
Clinician and Staff Perspectives on Participating in Practice-based Research (PBR): A Report from the Wisconsin Research and Education Network (WREN)
}

\author{
Amanda E. Hoffmann, MPH, Erin K. Leege, MPH, Mary Beth Plane, PhD, MSSW, \\ Katherine A. Judge, MSSW, Amy L. Irwin, MS, Regina M. Vidaver, PhD, and \\ David L. Hahn, MD, MS
}

Background: The success of practice-based research (PBR) depends on the willingness of clinicians and staff to incorporate meaningful and useful research protocols into already demanding clinic schedules. The impact of participation on those who implement multiple projects and how to address the issues that arise during this complex process remain incompletely described. This article reports a qualitative evaluation of the experiences of primary care clinicians and clinic staff who participated in multiple PBR projects with the Wisconsin Research and Education Network (WREN). Also included are their suggestions to researchers and clinicians for future collaborations.

Methods: For program evaluation purposes, WREN conducted 4 focus groups at its 2014 annual meeting. The main focus group question was, "How has participation in PBR affected you and your clinic?" A total of 27 project members from 13 clinics participated in 4 groups (physicians, nurses, managers, and other clinical staff). The 2 -hour sessions were recorded, transcribed, and analyzed to identify recurring themes.

Results: Five major focus group themes emerged: receptivity to research, outcomes as a result of participation, barriers to implementation, facilitators of success, and advice to researchers and colleagues. Focus group members find research valuable and enjoy participating in projects that are relevant to their practice, even though many barriers exist. They indicated that research participation produces clinical changes that they believe result in improved patient care. They offered ways to improve the research process, with particular emphasis on collaborative early planning, project development, and communication before, during, and after a project.

Conclusions: Clinics that participate in WREN projects remain willing to risk potential work constraints because of immediate or impending benefits to their clinical practice and/or patient population. Including a broader array of clinic personnel in the communication processes, especially in the development of relevant research ideas and planning for clinic implementation and ongoing participation in research projects, would address many of the barriers identified in implementing PBR. The themes and supporting quotes identified in this evaluation of WREN projects may inform researchers planning to collaborate with primary care clinics and clinicians and staff considering participating in research endeavors. (J Am Board Fam Med 2015;28:639-648.)

Keywords: Practice-based Research, Primary Health Care, Program Evaluation

Primary care practice-based research networks (PBRNs) develop and test clinical recommendations and organizational health care processes. ${ }^{1,2} \mathrm{~A}$ principal focus of these PBRNs is building trusting, collaborative relationships with clinics to foster a

This article was externally peer reviewed.

Submitted 28 January 2015; revised 3 June 2015; accepted 5 June 2015. mutual commitment to invest resources toward primary care research. Seen as "clinical laboratories for primary care research and dissemination," PBRNs are evolving into "learning communities, proving grounds for generalizable solutions to clin-

From the Department of Family Medicine and Community Health, University of Wisconsin School of Medicine \& Public Health, Madison, WI. 
ical problems, and engines for improvement of primary care delivery systems." ${ }^{3-5}$

Emerging evidence documents the overall experience $^{6-14}$ and outcomes ${ }^{15,16}$ of participating in practice-based research (PBR). Practicing clinicians, ${ }^{6-11}$ academic researchers, ${ }^{12}$ and experts in $\mathrm{PBR}^{14}$ have described their experiences in or made recommendations about implementing PBR. To assess direct experiences, Yawn et $\mathrm{al}^{13}$ used semistructured telephone interviews with 48 project nurses and physicians who participated in a PBR project around postpartum depression conducted in small to medium-sized family medicine clinics; 6 major themes emerged: (1) systematic approaches, (2) teamwork and communication, (3) spread to other conditions, (4) professional self-worth and recognition, (5) staff "expand" into new roles, and (6) research literacy. We were interested in determining whether similar themes would emerge from medium-sized to large practices participating in multiple PBR projects. We included managerial and programmatic staff, who we hypothesized might have different perspectives on $\mathrm{PBR}$ project involvement than clinical staff.

The Wisconsin Research and Education Network (WREN) is a state-wide PBRN that conducts a wide variety of projects, ranging from practice pattern assessment, quality improvement, and guideline dissemination up to and including randomized trials. ${ }^{17,18}$ WREN is housed within and supported by the University of Wisconsin School of Medicine and Public Health's Department of Family Medicine and Community Health and is also tightly integrated within the Community Academic Partnership core of the University of Wisconsin Institute for Clinical \& Translational Research, which is supported by the National Institutes of Health Clinical \& Translational Science Award program. ${ }^{17,18}$ During the 2014 WREN Convocation of Practices, we held focus groups to document the perspectives of clinicians, staff, and managers to inform us about WREN's current state. While our purpose was primarily program evaluation, we believe our

Funding: Funding for this project was provided by a grant from the Department of Family Medicine, University of Wisconsin School of Medicine \& Public Health, Madison, WI.

Conflict of interest: none declared.

Corresponding author: David L. Hahn, MD, MS, Wisconsin Research \& Education Network, Department of Family Medicine \& Community Health, 1100 Delaplaine Court, Madison, WI 53715 (E-mail: dlhahn@wisc.edu). results may be informative for other PBRNs in terms of themes, challenges, and responses to these challenges.

\section{Methods}

We conducted a systematic program evaluation at the 2014 WREN Convocation of Practices to determine how participation in the research process currently affects the work of WREN participants and clinics. The University of Wisconsin-Madison Health Sciences Institutional Review Board determined that the focus groups constituted program evaluation and were therefore exempt from institutional review board oversight.

\section{Recruitment}

We invited 53 individuals at 31 clinics with previous or current experience in WREN projects to participate in the focus groups, including physicians, advanced practitioners, clinic managers/directors, nurses, medical assistants, laboratory technicians, receptionists, and administrative staff to ensure a group reflecting the overall impact of WREN project participation on clinics. We asked for no more than 4 representatives from each clinic. As an incentive to participate, conference and lodging fees were waived for focus group participants.

\section{Focus Groups}

A total of 27 individuals from 13 clinics representing 11 health systems in rural, suburban, and urban areas throughout Wisconsin agreed to participate. Participants were assigned groups based on their clinic role: physicians $(\mathrm{n}=9)$, nurses $(\mathrm{n}=8)$, managers $(n=4)$, and other clinic staff $(n=6)$. Focus group members participated in a variety of WREN projects: public health disease surveillance, dissemination and implementation, workflow analysis, quality improvement, previsit planning, and chronic disease management. A trained master'slevel research coordinator and co-moderator facilitated each 2-hour session. The focusing question was, "How has participation in practice-based research affected you and/or your practice?" (Appendix Table 1).

\section{Analysis}

Focus group sessions were recorded and transcribed. Four authors reviewed each transcript. Three authors (AEH, EKL, MBP) reviewed all 4 
transcripts, whereas the fourth position rotated among the coauthors (KAJ, ALI, RMV, DLH). Transcripts were analyzed using the method of constant comparison, ${ }^{19}$ whereby readers coded individual units of information (open coding), grouped them into categories (axial coding), and finally developed themes and subthemes relevant to the data (selective coding). Each transcript was coded individually, with each author reading it ahead of time to identify broad themes, then meeting as a group to review each transcript line by line to decisively identify main themes, subthemes, and key quotes. Themes were compared within and between groups. Theoretical saturation was achieved when no new themes emerged during the fourth review of the transcript. Summary statements and key quotes from each group were combined to depict the suitability of themes based on recurrence. Statements then were collapsed further into succinct descriptions.

\section{Results}

Focus group members discussed their reactions to and the impact of at least 10 recent and ongoing WREN projects. Five major themes, each with 3 to 4 subthemes, emerged from the transcripts of all 4 groups: (1) receptivity to research; (2) outcomes as a result of participation; (3) barriers to implementation; (4) facilitators of success; and (5) advice to researchers and colleagues. There was remarkable consistency in responses, as all 4 groups' discussions contained all major themes. Each theme is discussed below in more detail, accompanied by illustrative quotes. The themes are further presented in Table 1 alongside current and future WREN approaches.

\section{Theme I: Receptivity to Research}

Focus group members revealed mostly positive feelings toward research in general, WREN staff, and WREN projects. They reported that participation in PBR enhanced teamwork, fostered new work relationships, and rejuvenated work life by providing professional satisfaction and allowing for continuous learning. Members enjoy knowing they can make meaningful contributions to their work and the health care field. For example, 2 physicians expressed the following: "What I've become fascinated in is how do we do what we do and why do we do it? And how could we do it better? Because we have now learned the muscle memory..." and "And kind of looking at it as . . how do I work in a more efficient way and give better care?"

Receptivity to research is enhanced when clinic staff become familiar with WREN, understand a particular project's purpose, and feel like they are partners in the process. Staff are willing to participate in PBR if project procedures respect the temporal demands of real-world clinical care, improve teamwork and patient care, and provide the clinic with a sense of ownership over the project. There are times when a clinic is not positioned to take on a project because of system or staffing issues, but they would be willing to work with researchers in the future.

\section{Theme II: Outcomes}

Each group shared examples of positive effects of research participation at both individual and clinic levels. For example, a nurse stated, “. . . it improves outcomes, WREN does. Practices do change because of their work." Focus group members explained that research has led to the incorporation of best practices into daily routine, with a manager explaining, "[participating is] a good way of just learning new skill sets that just become part of what you do."

\section{Improved Clinical Care}

Focus group members reported that patient encounters have been positively influenced by WREN participation. In addition to offering resources that led to improved patient education, some projects allowed practices to fill a clinical need by offering point-of-care testing, resulting in improved patient care and satisfaction. Given examples include speed of diagnosis, preventing costly follow-up, and reducing potential health system burden. A clinical staff member asserted, “. . . better care and service to our patients really should be a primary concern in health care. That is what we're there for." As a result of projects that incorporate patient perspectives or shared decision-making techniques, focus group members reported that communication between clinicians, clinic staff, and patients has improved, resulting in stronger relationships. In addition, a clinician provided specific examples, explaining, “. . . from a minimum standpoint, I think that my skills as a diagnostician improved in regards to diagnosing influenza and also the specific case of hypertension in young adults.” 
Table 1. Focus Group Themes Identified, Current Wisconsin Research and Education Network (WREN) Approaches and Future Opportunities

\begin{tabular}{lll}
\hline Focus Group Themes & Current WREN Approaches & Future Opportunities
\end{tabular}

\section{Receptivity}

To research in general

To WREN

To specific projects

II. Outcomes as a result of participation

Improved clinical care (eg, disease management, patient encounters) and system changes within the clinic (eg, developed process workflow)

Increased knowledge/awareness about:

Care management and research

Other clinics and networking

\section{Barriers}

Competing priorities

Time

System limitations
- WREN members are solicited for involvement in research projects and surveys throughout the year.

- Nonsystematic results are provided to participating clinics during and after the project.

WREN retains 5 clinics under contract that receive funding for a $20 \%$ FTE research support person at each site* made possible by University of Wisconsin Clinical \& Translational Science Award funding through the $\mathrm{NIH}{ }^{\dagger}$ Other clinics are compensated from specific grant funds.

Identify potential project sites via existing membership database and personal knowledge.

Anecdotal surveys/focus groups of WREN members to assess impact.

- Nonsystematic feedback of project results to clinics.

- Monthly WREN e-newsletter.

Highlight clinic accomplishments in WREN e-newsletter and at annual convocation.

Nonsystematic personal contacts with health system administrators and research directors to identify areas where PBR can provide enhanced implementation and/or rigorous program evaluation for health system clinical initiatives.

- Work with principal investigators to ensure WREN projects avoid requiring clinic staff to be "research personnel," eg, WREN staff, rather than clinic staff, perform patient consent processes (in person and remotely), data collection, and follow-up.

- Perform clinician and staff project education using short, repeated, validated, E-mailed educational "snippets" ("spaced education") that can be accessed at convenient times.

Prospectively identify the details of project participation so the clinic staff and administrators are fully informed of project expectations.
- Provide overviews of WREN and its research portfolio onsite at clinics throughout the state.

- Ensure principal investigators provide systematic and timely feedback of results to clinics.

- Improve relationships between WREN and health system administrators.

- Ensure that WREN is not a stranger to clinics when a research opportunity arises.

Survey nonmember clinics for research interests and capabilities.

More systematic post-project interviews to document sustainability and spread; may be nested within funded dissemination and implementation research.

- Systematic and rapid turnaround of results for every project.

- Provision of project results during onsite WREN overviews.

Develop WREN PBR participant mentoring program, where existing clinic participants are paired with new ones.

- Systematic evaluation of health systembased needs that PBR projects could inform.

- Meet with system administrators, managers, and staff in project planning stage to learn competing priorities at all levels.

- Make sure system administrators and managers are supportive of time requirements, and that staff know their time is protected.

- Prospectively identify low-burden, high-impact projects for PBR.

- Prospectively develop projects aligned with system priorities so PBR can assist clinic operations to rigorously assess the impact of health system initiatives to provide added value.

- Negotiate with clinic physicians, staff, managers, and system administrators regarding implementation process, and obtain (written) assurances that administrators and managers support the project's processes and methods.

- Develop formal arrangements with several integrated health systems on how best to propose and permit PBR. 
Table 1. Continued

\begin{tabular}{lll}
\hline Focus Group Themes & Current WREN Approaches & Future Opportunities \\
\hline Project implementation challenges & $\begin{array}{l}\text { - Prospectively identify the details of } \\
\text { project participation so the clinic staff and } \\
\text { administrators are fully informed of } \\
\text { project expectations. } \\
\text { - Perform site visits and practice } \\
\text { facilitation. }\end{array}$ & $\begin{array}{l}\text { Be a meaningful partner in project } \\
\text { initiation conversations-even for } \\
\text { multisite projects-to ensure there are } \\
\text { no surprises at the start of project } \\
\text { implementation. }\end{array}$ \\
&
\end{tabular}

\section{Facilitators}

Support from research coordinator

Additional incentives

Clinic staff

V. Advice to researchers

Project development/early planning

\section{Communication \\ Before project (eg, clear timeline and project expectations)}

During the project (eg, check-ins)

Following the project (eg, offer feedback and dissemination of results)

Get to know the clinic
WREN staff perform all project-related procedures (consenting, data collection, etc.) for most projects.

- Recognize participating clinics at annual meeting and via e-newsletters.

- Provide certificates of participation to clinics to display onsite.

- Provide CME credits for physicians.

WREN RRCs develop ongoing collegial relationships with clinic staff.

Open statements (online) to researchers that the best time to consult WREN is during formulation of the research question; the worst time to approach WREN is 2 weeks before the grant is due.

- Prospectively identify the details of project participation so the clinic staff and administrators are fully informed of project expectations.

- Identify any challenges to project initiation that need to be overcome.

WREN RRCs provide ongoing feedback through practice facilitation and informal E-mail and telephone conversations.

- Nonsystematic feedback of results to participating clinics.

- Publication of project results in the WREN monthly e-newsletter.

- WREN RRCs visit clinics on an asneeded basis depending on project requirements.

- WREN director and program manager selectively visit clinics and visit with CEOs, medical directors, clinicians and staff.
- Utilize more EHR-based outcome measures that unburden both clinic and research staff from data collection.

- Perform research to validate and to incorporate into usual care a variety of patient-reported outcome measures that (1) improve care and (2) can be used to assess outcomes for pragmatic trials.

- Provide template press releases to clinics regarding PBR participation and project outcomes.

- Educational credits for staff.

- Emphasize recertification for physicians.

Prospectively identify clinic managers and health system administrators, and develop collegial relationships with them.

- Prospectively seek out collaborations with researchers to promote the concept that PBR is a venue in which to perform patient-oriented research.

- Build in incentives integral to project design, eg, maintenance of certification, meaningful use, and alignment with medical home initiatives.

- Negotiate with clinic administrators, managers, and staff regarding the method of implementing project protocols.

- Schedule "talk back" teleconferences with participating sites before project initiation so they can elucidate their understanding of what they will be doing and how. Encourage group teleconferences so sites can learn from each other.

Develop specific protocols for communication during and after practice facilitation activities.

- Personal phone calls to clinic managers to relay information on the project results and the anticipated impact of incorporating the results into clinic activities.

- In-person meetings.

- Hold in-person meetings at the clinics to learn about what is important to the physicians and staff who work there, the issues they are facing that affect their current work, and how they think they can work with WREN on developing a protocol for an individual project.

- Provide overviews of WREN and its research portfolio onsite at clinics throughout the state.

*See http://www.fammed.wisc.edu/research/wren/about for details on the "Full Support Clinic" model.

'The "Community-Academic Partnership" program of the University of Wisconsin Institute for Clinical and Translational Research (ICTR).

CME, continuing medical education; EHR, electronic health record; FTE, full-time equivalent; NIH, National Institutes of Health; PBR, practice-based research; RRC, regional research coordinator; WREN, Wisconsin Research and Education Network. 
Many focus group members discussed how research participation has improved their care management, especially with chronic diseases. Clinical staff learned new ways to communicate diagnosis and care plans with patients. In addition, follow-up care improved as a result of a workflow redesign project, with one physician exclaiming, “. . . we've gotten more patients to come back!"

Focus group members who participated in a previsit planning study described a culture shift in their clinics that lasted beyond the study. The study protocol helped to improve habits to review records and identify issues before patient arrival, resulting in time saved in the long run. A clinical staff member stated, "I think [participation] empowered the [medical assistants] to speak up if they saw a patient that did not have the correct lab work or needed to come in, so I think it helped with the teamwork aspect of providing better care for our patients." One physician commented, "[I learned] the anatomy of the previsit. Not just the anatomy of the visit. The anatomy of the previsit, the expectations of the patients and then what goes into my decision making and how can that be improved."

\section{Systems Change}

Focus group members shared examples of system changes. For several clinics, documentation in the medical record improved (eg, flagging medication contraindications and reminders) because PBR participation showed clinicians the importance of consistency in creating accurate reports. Clinic staff learned how to pull the right kind of data and transform it into useful information. Participation led to the development of better patient panels and an understanding of population-level management wherein clinics could focus on patients with specific diseases in a more effective manner.

Successful workflow redesigns were frequently mentioned. Clinics have capitalized on staff strengths to improve internal processes and consistency across patient encounters. One physician expanded on this, stating, "I guess it is not how I do things, it is how I think about ... who would be the good resource for this at our clinic because... [we often forget] about that. It is like wait, they (staff) have great ideas, way better ideas than we (doctors) have because they are the ones doing it every day, day in and day out." Managers commented that after changes implemented during a recent WREN protocol, their clinic staff were better prepared to begin work on new systems requirements from their system's administration.

Last, participants cited a culture shift in the clinic to being more patient-centric. A clinical staff member expressed, "we all know if there's no patient, we do not exist. I mean, that is why we're here is for that patient. We're trying to get that culture."

\section{Knowledge/Awareness}

Both staff and physicians discussed how WREN participation promoted a sense of patients as partners, a feeling of belonging to a community of clinics, an awareness of one's clinic environment, and an increased appreciation for the role of research.

Several focus group members were surprised to discover that patients are receptive to research. One physician recalled, "I remember thinking the patients would be upset because [research] takes time ... I've been amazed at how few of them have ever refused. . . Most people have been very positive ... [and] very receptive of it. [They] actually think it is kind of cool to be a part of it . . When I first started doing it I thought 'well, I am going to get pushback,' but I really haven't." Working on WREN projects has taught clinics the importance of communicating with patients, the value of including patient perspective, and that patients want to be involved with their health care team. Focus group members explained that clinics must work with patients to address the issues that patients feel are most meaningful to their quality of life, realizing that not every patient expects to be "fixed" by their clinician.

Staff also gained a sense of validation and social support from collaborating with other clinics via research. Focus group members enjoyed learning about other clinics through WREN activities and conference attendance, explaining that it is nice to know they are "not alone" and other clinics face similar struggles. In addition, WREN projects allowed members to learn how their staff work together and what internal processes need improvements.

Last, focus group members explained that participation has taught them about the process of research, its importance in improving patient health, and that anyone can be involved. A clinical staff member recollected that ". . . before we did WREN, I did not know that there were research networks out there that were doing the practical 
value-added projects that WREN is doing and that help practices and clinicians in the operation side of the world."

\section{Theme III: Barriers}

Despite positive attitudes toward research, participants identified several barriers to PBR; limited time and competing clinical priorities were mentioned repeatedly. Others told of barriers as a result of being housed within a larger system, including administrators who state research is important but have difficulty facilitating its implementation, even on specifically approved projects. Some examples include difficulty obtaining approvals; not allocating enough staff time to do the work; and an inability to incorporate study procedures that differ from the clinic system's protocol. Implementation barriers include difficulty in always following study protocols, particularly with projects that do not fit into the clinic's workflow or that use procedures or testing that do not follow approved clinic protocol; and nonparticipating clinicians/staff who are unfamiliar with the study protocol and who are not always receptive or trained when having to fill in for project staff.

Each group reported frustrations relating to research, particularly with high-burden, lengthy studies that could contribute to staff burnout. Negative appraisals were mentioned when projects had an overwhelming amount of initial work and difficulties investing time without seeing immediate payback. A clinician also voiced that there is ". . . sort of a divide between clinicians and academics. And it is there. Nobody likes to talk about it, but I think that a lot of clinicians look down on academics for not being involved in actual care and academics look down on clinicians for not always providing best practices."

\section{Theme IV: Facilitators of Success}

All groups cited the importance of support from a research coordinator as a major facilitator of success. They value research coordinators' organizational skills, meeting and project facilitation, and general support to include responsive and positive feedback. One physician stated, "the human connection of the regional research coordinator is essential." A clinical staff member revealed, "initially it seemed like it was going to be overwhelming and it just was not. So a positive experience is just the interaction we had and the support we had to run the project. . . I would not hesitate if we were approached again." Likewise, focus group members acknowledged the significance of clinic staff in implementing projects, with one physician exclaiming, ". . . without our staff that study would have just crashed and burned." In addition, focus group members from full support clinics cited the importance of having a contract with WREN, because it allows protected time for clinic staff to complete assigned study responsibilities in addition to their normal workload. (See Table 1 for details on the WREN Full Support Clinic model.)

Other facilitators include incentives (eg, professional development credits, information-sharing at conferences, food at clinic meetings) and the feeling that research projects are partnerships between WREN and clinics. One manager said, "I like the concept that it feels more like a partnership than a top-down approach," further stating, "you can trust ... that the scientists that put it together are very thoughtful and they have done this before and you start seeing some themes in the type of work that it is. I think that is a nice fit for primary care."

\section{Theme V: Advice \\ Advice to Researchers}

Focus group members offered advice in response to many of the focus group questions. The following subthemes were identified: project development, familiarity with the clinic, and communication (before, during, and after study implementation).

Project Development. Participants across all groups stressed the importance of identifying and including all appropriate stakeholders (ie, patients, staff, clinicians, and administration) in the development phase. They suggest allowing clinicians and clinic staff time to provide feedback on study materials and protocols before implementation.

Familiarity with the Clinic. Building personal working relationships with clinics is necessary for both recruitment efforts and project implementation. To ensure success, it is important to match projects with clinic interests, priorities, workflows, resources, funding, philosophy, and timing. A clinical staff member expressed that ". . . a huge part of implementing these studies is WREN has to be able to adapt to us and our workloads and our staffing patterns and to have somebody in the WREN system ... spend the time to learn how [we] function and for us to have that contact." A 
manager advised that "... part of what WREN can do and what ideal research groups can do ... is to really do a critical look at the design and the fit [of the study] with the philosophy of the clinic, the resources of the clinic, [and] the funding."

Communication. Much advice centered on communication, both in general and during specific times throughout the project. Focus group members urged researchers to provide strong communication with everyone who will be involved in the project. They want clear, concise information about study expectations up front and throughout the project. They expect dissemination of results to the clinic following the project to learn from the project's outcomes. A physician described this further, stating, ". . . a closure to the loop would be useful. I think it would help to maintain enthusiasm for the projects. It would be good PR for enlisting for the next study."

In summary, WREN clinics desire involvement in all stages of the research process, from conception through implementation to dissemination.

\section{Advice to Colleagues}

Focus group members offered practical advice to their clinical colleagues contemplating PBR participation. One clinical staff member simply stated, "Go for it. Mainly they would learn so much from it and just to do it. Yes it is work, but you know, they can improve things, why not?" Many advised starting with a small project to "get one's feet wet." They reminded potential study participants to keep a positive attitude and open mind throughout the research process, have continuous engagement with all team members, and ask for help if needed. Another key step is to require WREN to provide as many study details as possible up front (eg, learn about WREN and the timeline, protocol, and scope of work). From the outset it is important to think of the big picture and what will happen after the study ends (ie, will the outcomes be usable in our clinic or transferable to our work?).

\section{Discussion}

This evaluation provided WREN with a rich source of information that will inform our future development. Knowledge of the context within which WREN operates is required to gauge the potential usefulness of this information to other PBRNs. Wisconsin has a tradition as a "group practice state" and is at the forefront nationally in terms of health care consolidation. ${ }^{20}$ WREN's experience may serve as an illustrative example for other PBRNs in terms of understanding the extent of the infrastructure needed to conduct PBR; how to deal with issues raised by health care consolidation, with its attendant loss of physician autonomy; and ways that researchers, clinic staff, patients, and other stakeholders can partner to develop and implement research that resonates with all stakeholders.

Several subthemes encountered herein (Table 1) that have been previously identified include (1) improved clinical care as a result of learning and adapting best practices, ${ }^{6-8}$ (2) improved systematic approaches, ${ }^{8,13}$ (3) more effective teamwork and communication, ${ }^{13}$ (4) increased professional selfworth, ${ }^{13}$ and (5) opportunity and support for staff members to "expand" into new roles. ${ }^{13}$ Previously identified barriers include (1) excessive time and resources needed to conduct a project ${ }^{9,21,22}$ and (2) difficulty adapting new protocols alongside or in place of existing protocols. ${ }^{7}$

Additional clinic team comments and themes not previously emphasized in the literature include (1) the need for clinic physicians, staff, patients, and administrators to be part of the planning process and (2) barriers posed by health care consolidation that represent an increasing challenge to PBRN activities nationally. Health care consolidation means that more clinics will become part of larger systems; therefore, we anticipate that other PBRNs will be increasingly challenged to function effectively in this environment. We anticipate the observations presented here have the potential to generally inform PBRN activity planning and implementation. We identify potential responses to the various challenges identified by the focus groups in Table 1 .

\section{Limitations}

Before the start of the focus groups, all conference attendees listened to a plenary session presented by a WREN clinician, "How Participation in PBRNs has Improved my Clinical Practice." Consequently, focus group discussions may have been influenced by what participants heard during this presentation. While the title of the speaker's talk was positive, he nonetheless devoted a significant amount of his presentation to describing one high-burden study. The focus groups comprised volunteers who at- 
tended the 2014 WREN Convocation and who had participated in at least 1 previous WREN project. The attitudes and experiences of this group are unlikely to be generalizable to nonparticipating clinics. Information gleaned from this group of highly motivated individuals may allow us to encourage others to participate. WREN research coordinators, who had ongoing collegial relationships with the focus group participants, facilitated the focus groups. As such, participants may have felt the need to temper feedback that could potentially harm the working relationship with the research coordinator. To mitigate this limitation, the facilitators' script encouraged participants to share any and all feedback, including negative responses.

\section{Conclusions}

PBRNs rely on primary care clinics that are capable of being fully engaged in the implementation and management processes of ongoing research projects. Understanding clinician and staff experiences with PBR is necessary to maintain an effective collaborative partnership between academic researchers and the clinic staff who are willing to complete this type of work alongside their usual clinical responsibilities. The evaluative process we undertook may inform (1) researchers planning to study patients or processes in primary care clinics, (2) funding organizations supporting PBR, and (3) clinicians and staff who are considering participating in research endeavors.

The authors acknowledge the WREN members who gave time to share their perspectives. We also thank Jon Temte, MD, MS, $\mathrm{PhD}$, and John Beasley, $\mathrm{MD}$, for their careful review of this manuscript.

\section{References}

1. Primary care practice-based research networks. Rockville, MD: Agency for Health care Research and Quality. December 2012. Available from: http://www.ahrq.gov/ research/findings/factsheets/primary/pbrn/index.html. Accessed January 28, 2015.

2. Nutting PA, Beasley JW, Werner JJ. Practice-based research networks answer primary care questions. JAMA 1999;281:686-8.

3. Westfall JM, Mold J, Fagnan L. Practice-based research-"Blue Highways" on the NIH roadmap. JAMA 2007;297:403-6.

4. Heintzman J, Gold R, Krist A, Crosson J, Likumahuwa S, DeVoe JE. Practice-based research networks (PBRNs) are promising laboratories for con- ducting dissemination and implementation research. J Am Board Fam Med 2014;27:759-62.

5. Mold JW, Peterson KA. Primary care practice-based research networks: working at the interface between research and quality improvement. Ann Fam Med 2005;3(Suppl 1):S12-20.

6. Rhyne R, Sussman AL, Fernald D, et al. Reports of persistent change in the clinical encounter following research participation: a report from the Primary Care Multiethnic Network (PRIME Net). J Am Board Fam Med 2011;24:496-502.

7. Curro FA, Thompson VP, Grill A, et al. An assessment of the perceived benefits and challenges of participating in a practice-based research network. Prim Dent J 2012;1:50-7.

8. Fagnan LJ, Handley MA, Rollins N, Mold J. Voices from left of the dial: reflections of practice-based researchers. J Am Board Fam Med 2010;23:442-51.

9. Plane MB, Beasley JW, Wiesen P, McBride P, Underbakke G. Physician attitudes toward research study participation: a focus group. WMJ 1998;97: 49-51.

10. Wallin L, Bostrom AM, Wikblad K, Ewald U. Sustainability in changing clinical practice promotes evidence-based nursing care. J Adv Nurs 2003;41:509-18.

11. Bakken S, Lantigua RA, Busacca LV, Bigger JT. Barriers, enablers and incentives for research participation: a report from the Ambulatory Care Research Network (ACRN). J Am Board Fam Med 2009;22: 436-45.

12. Yawn BP, Dietrich A, Graham D, et al. Preventing the voltage drop: keeping practice-based research network (PBRN) practices engaged in studies. J Am Board Fam Med 2014;27:123-35.

13. Yawn BP, Pace W, Dietrich A, et al. Practice benefit from participating in a Practice-based Research Network study of postpartum depression: a National Research Network (NRN) report. The J Am Board Fam Med 2010;23:455-64.

14. Dolor RJ, Schmit KM, Graham DG, Fox CH, Baldwin LM. Guidance for researchers developing and conducting clinical trials in practice-based research networks (PBRNs). J Am Board Fam Med 2014;27: 750-8.

15. Sinclair-Lian N, Rhyne RL, Alexander SH, Williams RL. Practice-based research network membership is associated with retention of clinicians in underserved communities: a Research Involving Outpatient Settings Network (RIOS Net) study. J Am Board Fam Med 2008;21:353-5.

16. Abraham AJ, Knudsen HK, Roman PM. The relationship between Clinical Trial Network protocol involvement and quality of substance use disorder treatment. J Subst Abuse Treat 2014;46:232-7.

17. Wisconsin Research \& Education Network. About WREN. Last updated December 1, 2014. http:// 
www.fammed.wisc.edu/research/wren/about. Accessed January 28, 2015.

18. Beasley JW, Cox NS, Livingston BT, et al. Development and operation of the Wisconsin Research Network. Wis Med J 1991;90:531-2, 534-7.

19. Onwuegbuzie AJ, Dickinson WB, Leech NL, Zoran AG. A qualitative framework for collecting and analyzing data in focus group research. Int $\mathrm{J}$ Qual Methods 2009;8:1-21.

20. Boulton, G. Wave of consolidation engulfing health care systems. Milwaukee Journal Sentinel. April 5,
2015. Available from: http://www.jsonline.com/ business/wave-of-consolidation-engulfing-healthcare-systems-b99474527z1-298731631.html. Accessed July 22, 2015.

21. Temte JL, Beasley JW. Rate of case reporting, physician compliance, and practice volume in a practicebased research network study. J Fam Pract 2001;50: 977.

22. Temte JL, Hunter PH, Beasley JW. Factors associated with research interest and activity during family practice residency. Fam Med 1994;26:93-7.

\section{Appendix Table 1. Focus Groups held at Wisconsin Research and Education Network's (WREN's) 2014 Convocation of Practices: Introductory, Transition, and Key Focus Group Questions}

\begin{tabular}{|c|c|c|}
\hline Question & Follow-up Questions & Question Type \\
\hline $\begin{array}{l}\text { 1. How did you come to be a part of the WREN } \\
\text { studies at your clinic?* }\end{array}$ & $\begin{array}{l}\text { a. What was your role in working with the WREN } \\
\text { projects at your clinic? }\end{array}$ & Introductory \\
\hline $\begin{array}{l}\text { 2. What do people in your clinic say when they } \\
\text { talk about WREN studies?* }\end{array}$ & & Transition \\
\hline $\begin{array}{l}\text { 3. Did participation in WREN studies affect the } \\
\text { way you do your work in the clinic? }\end{array}$ & a. Are there any other examples? & Key \\
\hline $\begin{array}{l}\text { 4. Can you describe something positive that has } \\
\text { happened because you were a part of a WREN } \\
\text { study? }\end{array}$ & & Key \\
\hline $\begin{array}{l}\text { 5. Can you describe something negative that has } \\
\text { happened as a result of being a part of a WREN } \\
\text { study? }\end{array}$ & $\begin{array}{l}\text { a. If yes, what do you think contributed to this } \\
\text { negative? } \\
\text { b. Can you think of any way we could have turned } \\
\text { this situation around into a positive? }\end{array}$ & Key \\
\hline $\begin{array}{l}\text { 6. What do you consider to be the most important } \\
\text { things you learned as a result of your } \\
\text { participation in WREN studies? }\end{array}$ & & Key \\
\hline $\begin{array}{l}\text { 7. Is there anything that made your participation } \\
\text { in WREN studies easier? }\end{array}$ & & Key \\
\hline $\begin{array}{l}\text { 8. Is there anything that made your participation } \\
\text { in WREN studies more difficult? }\end{array}$ & & Key \\
\hline $\begin{array}{l}\text { 9. What can WREN do to support clinic staff } \\
\text { during a WREN project? }\end{array}$ & & Key \\
\hline $\begin{array}{l}\text { 10. What advice would you have for someone who } \\
\text { wants to do a project with your clinic? }\end{array}$ & & Ending \\
\hline $\begin{array}{l}\text { 11. If you were giving advice to a colleague about } \\
\text { working with WREN, what would you say? }\end{array}$ & & Ending \\
\hline $\begin{array}{l}\text { 12. Is there anything we have not yet discussed } \\
\text { that you think is important for us to know? }\end{array}$ & & Ending \\
\hline
\end{tabular}

*Questions asked in a round-robin style. Unless indicated, remaining questions used a "popcorn" style to generate ideas through rapid-fire responses. Prompts were provided when a question did not generate any initial responses.

${ }^{\dagger}$ Participants wrote individual responses on sticky notes and shared by placing then on a wall, organizing by similar ideas. 